\title{
Educación escolar
}

School Education / Educação Escolar

Adolescentes y ocio: desarrollo positivo y transición hacia la vida adulta

Teenagers and Leisure: Positive Development and Transition to Adulthood

Adolescentes e lazer: desenvolvimento positivo e transição à vida adulta

Aránzazu Albertos, Alfonso Osorio, Carlos Beltramo

Enseñanzas y aprendizajes sobre la Cátedra de la paz en Colombia Lessons and Takeaways from the Lecture on Peace in Colombia

Ensino e aprendizagem sobre a "cadeira da paz" na Colômbia

Catalina María Vásquez-Russi 



\section{Adolescentes y ocio: desarrollo positivo y transición hacia la vida adulta}

\section{Aránzazu Albertos}

https://orcid.org/000o-0002-3590-8364 Universidad de Navarra, España aalbertos@unav.es

\section{Alfonso Osorio}

https://orcid.org/oooo-0003-4337-5382 Universidad de Navarra, España aosorio@unav.es

\section{Carlos Beltramo}

https://orcid.org/oooo-0oo1-8382-8134

Universidad de Navarra, España

cbeltramo@unav.es

\section{Resumen}

A partir del siglo XX el ocio ha tenido un papel crucial en la sociedad, la economía, la cultura y la educación. Este hecho ha despertado un gran interés por estudiar la conexión que tiene este fenómeno con los estilos de vida, la satisfacción, el entorno, y la relación entre ocio y trabajo. Este artículo analiza la influencia del ocio en la vida de los jóvenes. En la primera parte, se realiza una revisión bibliográfica de las principales teorías sociológicas y psicológicas que explican su sentido, evolución y relación con el trabajo. En la segunda parte, se expone el modelo de desarrollo positivo, centrado más en el florecimiento del potencial de los jóvenes que en prevenir conductas de riesgo. El uso positivo del tiempo libre se basa en la realización de actividades estructuradas, con participación voluntaria, motivación intrínseca y supervisadas por adultos. Se concluye que la implicación de los jóvenes en asociaciones, organizaciones e instituciones que desarrollen actividades estructuradas de participación voluntaria o autoimplicada facilita la transición a la vida adulta, en relación con el uso constructivo del tiempo unido a un ocio libre y placentero.

\section{Palabras clave (Fuente: tesauro de la Unesco)}

Adolescencia; desarrollo de la personalidad; educación juvenil; juventud; ocio; prevención de conductas de riesgo; tiempo libre.

Recepción: 14/11/2019 | Envío a pares: 05/03/2020 | Aceptación por pares: 20/03/2020 | Aprobación: 06/04/2020 


\title{
Teenagers and Leisure: Positive Development and Transition to Adulthood
}

\begin{abstract}
Since the $20^{\text {th }}$ century, leisure has had a crucial role in society, the economy, culture, and education. This has aroused great interest in studying the connection between this phenomenon and lifestyles, satisfaction, the environment, and the leisure-work relationship. This article analyzes the influence of leisure on the lives of young people. The first part contains a literature review of the main sociological and psychological theories that explain its meaning, evolution, and relationship with work. The second part presents the positive development model, focused on unlocking young people's potential rather than on preventing risky behaviors. The positive use of free time relies on structured, voluntary, intrinsically motivated, adult-supervised activities. In brief, the involvement of young people in associations, organizations, and institutions that carry out structured, voluntary activities facilitates the transition to adulthood concerning the constructive use of time and free, pleasant leisure.
\end{abstract}

\footnotetext{
Keywords (Source: Unesco thesaurus)

Adolescence; personality development; youth education; youth; leisure; prevention of risk behaviors; recreation.
} 


\section{Adolescentes e lazer: desenvolvimento positivo e transição à vida adulta}

\section{Resumo}

Desde o século XX, o lazer vem desempenhando papel crucial na sociedade, na economia, na cultura e na educação. Isso desperta um grande interesse em estudar a conexão que esse fenômeno tem com os estilos de vida, a satisfação, o entorno e a relação entre lazer e trabalho. Este artigo analisa a influência do lazer na vida dos jovens. Na primeira parte, é realizada uma revisão bibliográfica das principais teorias sociológicas e psicológicas que explicam seu sentido, evolução e relação com o trabalho. Na segunda, é exposto o modelo de desenvolvimento positivo, centralizado mais no florescimento do potencial dos jovens do que no prevenir comportamentos de risco. O uso positivo do tempo livre está baseado na realização de atividades estruturadas, com participação voluntária, motivação intrínseca e supervisionadas por adultos. Conclui-se que o envolvimento dos jovens em associações, organizações e instituições que desenvolvem atividades estruturadas de participação voluntária ou autoimplicada facilitam a transição à vida adulta, no que diz respeito ao uso construtivo do tempo unido a um lazer livre e prazeroso.

\section{Palavras-chave (Fonte: tesauro da Unesco)}

Adolescência; desenvolvimento da personalidade; educação de jovens; juventude; lazer; prevenção de comportamentos de risco; tempo livre. 


\section{Concepto e historia del ocio}

Para entender el papel y el sentido del ocio en la actualidad es necesario referirse a su evolución cultural e histórica. El primer término que se utiliza para denominar ocio fue skholé, que apareció en Grecia y significa paz, estudio o escuela y hace referencia a actividades no productivas, focalizado en la formación y desarrollo de la persona (De Grazia, 1966). En Roma el sentido del ocio evoluciona hacia un tiempo de no trabajo que facilita el descanso y la recreación y prepara para emprenderlo. El ocio (otium) tiene como finalidad el negotium (ocupación, trabajo) y ambos forman parte de una vida unitaria (André, 1966). En el periodo que abarca desde la Baja Edad Media hasta la Revolución Francesa se da una separación, cada vez más dilatada, entre ocio y trabajo. El ocio es señal de distinción, por oposición al trabajo, y hay una intención de minimizar este último (Veblen, 2005).

La influencia de la llustración se manifiesta en que el ocio se aleja progresivamente del ideal de contemplación griego y va adquiriendo fuerza la idea de trabajo como expresión máxima del hombre, de la confianza en sí mismo. El ritmo de trabajo se hace más duro. Los burgueses, máximos exponentes de la moral del esfuerzo, dedican su vida al trabajo o a ocios instructivos (Cacérès, 1973). La revolución industrial favorece la polarización de la dicotomía entre tiempo de trabajo y tiempo de descanso (Weber, 2012).

\section{El origen de los estudios sobre ocio}

La sociología del ocio nace en la primera mitad del siglo XX, con un abanico de autores, entre los que destacan: Lynd y Lynd (1929), que hablan de la importancia que tiene sobre el rendimiento en el trabajo el comportamiento en el tiempo libre; Mead (1957) y Wolfenstein (1955), quienes afirman que el tiempo libre se convierte en un tiempo de consumo personal y evidencian el paso de una moral de sacrificio a una de placer.
Es a partir de Riesman cuando se impulsa la reflexión sobre el ocio y la creación, en 1955, ya con un centro dedicado a la investigación sobre el ocio. En su obra más conocida, The Lonely Crowd, junto con Denney y Glazer (1961), Riesman delinea un nuevo tipo humano, creado por las sociedades de la comunicación y del consumo. Como conclusión, este ensayo afirma que el hombre ya no se realiza con el trabajo, postura que cambiará en su segunda obra, Abundance for what? (1964), en la que defiende la necesidad de volver al trabajo como fuente de realización personal. En cualquier caso, lo que queda confirmado en estos estudios es el valor económico que adquiere el ocio.

La postura pesimista que mantiene Riesman en su primera obra se ve confirmada por los análisis de otros autores más o menos contemporáneos -como Mannheim (1953), de la Escuela de Frankfurt, y Mills (1951)- que insisten en el carácter alienante y despersonalizado del ocio en la sociedad del siglo $X X$. Debido a la difusión de subproductos culturales y a su consumo masivo, esto genera pasividad, conformismo y la pérdida de libertad, espontaneidad y creatividad. En una línea diferente, a principios de los años sesenta, Anderson (1961), Kaplan (1960) y Havighurst (1961) destacan el carácter subjetivo o personal que presenta al ocio, actividad que se define por la satisfacción que se obtiene de ella y porque favorece la buena adaptación social.

\section{La sociología del ocio en Europa}

La preocupación por el ocio y el estudio de las condiciones en que se produce comienza en Europa con retraso respecto de los Estados Unidos. Los autores más relevantes son Friedmann y Dumazedier. Para Friedman (1956), la interrelación entre ocio y trabajo se puede leer en el sentido de que todo aquello que se vive durante el tiempo dedicado al ocio repercute en diversas formas en el trabajo, sobre todo los valores que ha creado con sus actividades de ocio. Como consecuencia, es conveniente 
buscar ocios libres y activos e insistir en que las instituciones públicas los potencien. Su obra ha influido notablemente en la realización de análisis sobre las relaciones entre trabajo y ocio. Más adelante Friedmann (1963) define el ocio como una actividad libre y gratuita que aporta satisfacción inmediata.

Para Dumazedier (1964), el ocio es el conjunto de ocupaciones que realiza el individuo de manera voluntaria, que se caracterizan por tres "d": descanso, diversión y desarrollo, este último referido tanto a la formación como a la información. Según este autor, el ocio es un factor de cambio y de evolución social y es necesario hacer del ocio un tiempo de educación. Para llevar a cabo este cambio es fundamental que los poderes públicos determinen políticas completas y coherentes de ocio.

Según Cuenca y Goytia (2012), en torno a los años 60 existe un amplio acuerdo entre los investigadores respecto a las tres líneas principales de definición del ocio: tiempo, actividad y actitud, considerándose esta última como estado mental o ex periencia subjetiva. La investigación en estos años se centra en las prácticas y en el uso del tiempo y de ella se sustrae una definición objetivista del ocio, como tiempo libre o conjunto de actividades practicadas en el tiempo liberado de obligaciones.

\section{La perspectiva psicológica del ocio}

En torno a los años 70 se da un giro y los estudios sobre el ocio se centran en la perspectiva psicológica del desarrollo de la personalidad. Este nuevo enfoque lo inicia Neulinger (1974) con su libro The psychology of leisure. Este autor centra su interés en la evaluación de la personalidad a través del ocio, porque es el lugar ideal para investigar la dinámica de la personalidad según cinco dimensiones: libertad percibida, motivación intrínseca, motivación extrínseca, objetivo final y objetivo instrumental. De estas características cabe destacar que la motivación intrínseca hace referencia al ocio clásico, donde la actividad se realizaba por sí misma.
Para Neulinger es necesaria la promoción del ocio mismo como elemento de autorrealización humana, junto con la formación de actitudes de ocio dentro y fuera del trabajo, hasta crear una atmósfera que equipare el valor del ocio al del trabajo. En sus estudios, el trabajo no es un objeto de estudio independiente, ni es lo opuesto del ocio: existe una conexión entre ellos. Siguiendo esta línea de investigación, Iso Ahola (1980) publica el primer libro de texto sobre la materia, proponiendo la libertad percibida y la motivación intrínseca como condiciones fundamentales para que el sujeto experimente la vivencia del ocio.

Csikszentmihalyi $(1975 ; 1996)$ desarrolla el concepto de flow o experiencia óptima, según el cual se da el equilibrio que el sujeto necesita entre el reto de la actividad y su destreza. El desarrollo óptimo es la integración de todos los aspectos de la vida, llenando creativamente el tiempo libre y aprendiendo a disfrutar de lo que se está haciendo, tanto si se llama trabajo como si se llama ocio. Respecto al desarrollo personal, Csikszentmihalyi (2001) afirma que, para beneficiarse de un desarrollo óptimo, la persona debe aprender a aumentar su complejidad psicológica en el tiempo libre y en sus tareas obligatorias.

Tinsley y Tinsley (1986) proponen un modelo holístico de estudio del ocio, que enfatiza la importancia de centrarse en la experiencia subjetiva, más que en las actividades en sí mismas. Esta nueva perspectiva psicológica trata de aprehender y categorizar la percepción del sujeto, considerando el ocio como una experiencia preparada por ciertas condiciones, dotada de determinadas cualidades y beneficios. Las actitudes del trabajo podrían satisfacer los siete atributos del ocio: los que proceden de la experiencia subjetiva (concentración, olvido de sí mismo y disminución de la conciencia de tiempo) y de la experiencia afectiva (sentimiento de libertad; percepción enriquecida de objetos y eventos; aumento de la intensidad de las emociones y sensibilidad corporal de las emociones), y pueden añadirse 
cuatro características más: libremente elegido, intrínsecamente satisfactorio, estímulo óptimo y sentido del compromiso.

\section{Estudios contemporáneos sobre ocio}

En los años 90 los estudios de ocio se centran en la experiencia, también llamada vivencia. El referente más importante es la "Carta sobre la educación del ocio" (WLRA, 1993) fruto del Congreso Mundial sobre Ocio de la World Leisure and Recreation Association, donde el ocio se define como un área específica de la experiencia humana -con sus beneficios propios, entre los cuales están la libertad de elección, la creatividad, la satisfacción, el disfrute, el placer y una mayor felicidad- que comprende expresiones y actividades de naturaleza física, intelectual, social, artística o espiritual.

Según Cuenca y Goytia (2012), a partir de los años 2000 la visión del ocio se amplía y se complejiza, pues se estudia la naturaleza multidimensional, multifase y dinámica del ocio como experiencia. En lo referente a la relación entre ocio y trabajo, hay que destacar las aportaciones de dos autores. Stebbins, al acuñar el concepto de ocio serio (1982), manifiesta que hay actividades que permiten ejercitar las habilidades artesanales y las actividades creativas, con tal implicación perfeccionista que el producto final se aproxima a la calidad artesanal (Stebbins, 2012); por su parte, Rifkin (1995) afirma que la solución a la crisis del trabajo consiste en sustituir el valor de la acumulación de lo material por el valor de la vivencia del ocio. Desde el punto de vista educativo, propone enfocar la vida desde el ser y no desde el tener.

\section{La articulación de las teorías del ocio}

Las teorías mencionadas han sido significativas en la elaboración de un marco explicativo para el ocio. Los estudios sobre el comportamiento en el ocio y tiempo libre comenzaron en Estados Unidos y en Canadá, debido a la demanda servicios de ocio, flexibles y coordinados. Estos estudios continuados se centraron en los deseos y necesidades percibidas en los clientes de actividades recreativas, y en el estudio de conceptos y teorías comportamentales que sirvieran para la mejora de los servicios que prestan organismos y entidades (Harré, 1990; Langenhove, 1992).

La psicología social, que ha tomado un papel relevante en el análisis del fenómeno del ocio, ha focalizado sus estudios en dos terrenos: el contexto en el que se desarrolla (por ejemplo, el turismo) (Riesman, 1968) y el que se refiere al consumo, uso de nuevas tecnologías o prácticas deportivas (Baudrillard, 2009), camino iniciado por Neulinger (1974).

Rodríguez y Agulló (2002) afirman que es necesaria una articulación entre la psicología social y los estudios de ocio, y añaden como factores relevantes los que se relacionan con los estilos de vida, la satisfacción, la relación entre ocio y trabajo, y los comportamientos de ocio que generan, o pueden generar, problemas en el individuo o en el entorno. Estos autores denominan a este tipo de ocio problemático "ocio desviado". Para Codina (2007), el ocio es un objeto de estudio complejo o, más bien, "borroso". Según esta autora, los estudios de ocio han experimentado una psicologización centrada en el método de la psicología social, para la que las actividades de ocio (de tipo cultural, deportivas o con amigos) constituyen fenómenos de influencia social, motivo por el que esta disciplina ha tomado una posición central en las ciencias del ocio.

A modo de síntesis, se puede decir que autores como Dumazedier (1971) y Munné (1980) coinciden en resaltar la complejidad del ocio. Para Munné, el ocio tiene un significado indiscutible y un valor psicológico, a la vez que participa en un entramado de problemas de orden sociocultural y de sentido antropológico; esto explica el interés que despierta tanto en la sociología como en la psicología social. Como afirma Romo (2003), las cuestiones que rodean el tiempo libre son un campo para investigación científica y un lugar de reflexión política, económica y social, que deriva de la discusión sobre el modelo de desarrollo que se tiene y al que se aspira. 


\section{El ocio y los adolescentes}

\section{Caracteristicas identitarias de los adolescentes}

A los avances citados de la psicología social y la sociología sobre el ocio han de sumarse otras teorías significativas que sirven de base conceptual y explicativa de las características identitarias y evolutivas de la etapa adolescente. Erikson (1968), a través de su teoría sobre el desarrollo psicosocial de los adolescentes, afirma que están en una etapa cuya tarea fundamental consiste en alcanzar su identidad y resolver sus propias crisis, para convertirse en un adulto único, con sentido coherente del yo y un papel valorado en la sociedad. Este autor afirma que los adolescentes no forman su identidad tomando como modelo a otros, como hacen los niños, sino que hacen una sintesis de identificaciones anteriores y crean una nueva estructura psíquica, que es mayor que las partes que la forman (Kroger, 1993).

Para formar su identidad, los adolescentes deben establecer y organizar sus habilidades, necesidades, intereses y deseos, de forma que puedan ser expresados en un contexto social. En esta etapa se da una "moratoria psicosocial" (Erikson, 1968), en la cual el adolescente tiene una intensa interacción con el entorno, como si fuera un campo de pruebas (personas, objetos o sentimientos), y aplaza las consecuencias de sus acciones. La identidad se forma en la medida en que los jóvenes resuelven tres problemas principales: la elección de ocupación, la adopción de valores en los que creer y por los que vivir y el desarrollo de una identidad sexual satisfactoria.

Según Bernal y Köning (2017), el contexto y el ambiente en los que se desarrollan los procesos educativos tienen una relevancia especial, por ser el crisol en el que se forman las relaciones interpersonales que forjan su identidad. El estudio realizado por estos autores revela que un buen funcionamiento familiar y una buena relación humana en el aula favorecen un proceso educativo de calidad.

\section{El desarrollo positivo adolescente}

Generalmente la visión de la adolescencia presenta rasgos dramáticos, en los que enfatizan numerosas investigaciones. Existe una preocupación por lo que Puig y Trilla denominan "ocio nocivo" (1987, p. 94) y Rodríguez y Agulló (2002, p. 130) "ocio desviado". Esta visión negativa ha sido cuestionada y reformulada por Coleman (1978) y Larson (2000). Este último, en un estudio sobre el tiempo libre en la adolescencia, hace referencia a la cantidad de jóvenes que parecen estar aburridos, desmotivados y sin entusiasmo vital. Estos niveles de aburrimiento y pasividad no parecen ser indicadores de psicopatologías, sino más bien signos de deficiencia en el desarrollo positivo. Este autor señala, como un factor crucial para el desarrollo de los adolescentes, que se involucren en su tiempo libre en actividades que los entusiasmen y promuevan el desarrollo de habilidades necesarias para su vida.

Para Oliva y Pertegal (2015), aunque hay que valorar positivamente los programas de prevención de conductas de riesgo, es necesario pasar de un modelo de prevención, basado en el déficit, a un modelo de desarrollo positivo, que promueva la competencia personal, esté centrado en el bienestar, ponga énfasis en la existencia de condiciones saludables y expanda el modelo de salud, hasta incluir las habilidades sociales, conductas y competencias necesarias para tener éxito en la vida social, académica y profesional. Esta propuesta, en la línea con los modelos sistémicos, se basa en la idea de que todo adolescente es un ser plástico que tiene un potencial para un desarrollo exitoso y saludable, y ese potencial se desarrolla a nivel personal en su interacción con su entorno (Lerner, 2006). El eje de este modelo es el desarrollo de competencias, que son "las capacidades de los adolescentes para generar respuestas flexibles y adaptativas ante las demandas y tareas evolutivas que deben afrontar a partir de la pubertad" (Oliva y Pertegal, 2015, p. 24).

Damon (2009) propone como aportación relevante para el desarrollo el concepto de "propósito de 
vida", definido como una intención estable y generalizada de conseguir algo que es significativo para el adolescente, a la vez que tiene consecuencias en el mundo que lo rodea. En este propósito se puede incluir una filosofía con cierta transcendencia, unas acciones o planes de futuro, una significatividad para el sujeto y una inclusión de este propósito en la identidad personal. Todo esto se puede traducir en el logro académico, el arte, el liderazgo, la religión o el servicio a la comunidad. Los adolescentes que manifiestan un claro propósito vital suelen mostrar mejores estrategias para afrontar situaciones generadoras de estrés y también presentan mayor cohesión psicológica, lo que significa que sustentan una serie de valores como la humildad, la integridad y la vitalidad que dan consistencia a su desarrollo moral y personal, pudiendo, incluso, hablarse de la espiritualidad como ingrediente del propósito de vida.

Otro concepto importante, considerado por Larson (2000) como el núcleo del desarrollo positivo adolescente, es la "iniciativa personal". Este concepto se puede definir como la capacidad para tener una motivación intrínseca y dirigir la atención y el esfuerzo hacia un objetivo que suponga un reto personal; además es un requisito para el desarrollo de otras competencias como la creatividad, el liderazgo, el altruismo o la conducta cívica. Para este autor son tres los elementos clave de la iniciativa: la motivación intrínseca; el compromiso, la atención y el esfuerzo; y la continuidad durante un periodo largo. En el contexto escolar no están presentes estos tres elementos; en cambio, en el contexto del tiempo libre, las actividades estructuradas (actividades organizadas por adultos) tienen como efecto el desarrollo de la iniciativa, ya que combinan la motivación intrínseca, la concentración y la duración del tiempo.

Al respecto, Lerner (2004) propone como indicadores del desarrollo positivo adolescente, que deben aparecer en cualquier programa, cinco competencias, a las que denomina las "cinco ces": la competencia, habilidad de comportarse de manera eficaz y adecuada en las relaciones interpersona- les, en distintos contextos y en el desempeño de actividades; la confianza en sí mismo; la conexión con personas significativas en distintos entornos; el carácter, como integridad personal, respeto y asunción de normas sociales que llevan al ajuste del comportamiento propio a las pautas adecuadas; y el cuidado y la compasión, como empatía y solidaridad con los otros.

Algunos autores defienden que el "modelo salutogénico" facilita las claves para el óptimo desarrollo de la salud y, por extensión, de la persona, en concreto, de los adolescentes (Rivera et al., 2011). Este modelo se centra en lo que Antonovsky (1993a) llamó "sentido de coherencia" o sense of coherence (SOC), que se define como una orientación global de quien posee un sentimiento de confianza integral y persistente, basada en: estímulos provenientes de entornos internos y externos de la persona que son estructurados, predecibles y explicables; recursos disponibles para reconocer las demandas de dichos estímulos; demandas que constituyen desafíos merecedores de inversión y compromiso (Antonovsky, 1987, p. 107). Según este autor, el SOC estaría compuesto por tres elementos: la comprensibilidad (componente cognitivo), la manejabilidad (componente instrumental o de comportamiento) y la significatividad (componente motivacional). El SOC integra, por un lado, la capacidad del sujeto para comprender cómo está organizada su vida y cómo se sitúa él frente al mundo; por otro, la capacidad de manejarla; y, finalmente, el sentimiento de que tiene sentido, que la propia vida está orientada hacia metas que desea alcanzar. Según Rivera et al. (2011) el modelo salutogénico coincide con la teoría del capital social (Devine y Parr, 2008; Glover y Hemingway, 2005; Portes, 1998) en la que el "asociacionismo juvenil” es el mejor recurso para el desarrollo de los adolescentes.

En síntesis, el modelo de la competencia mejora el enfoque tradicional de déficit, otorgando además una importancia clave a las fortalezas del carácter y a los recursos activos, tanto a nivel personal 
como del contexto, con lo que realza la perspectiva evolutivo-sistémica que caracteriza el desarrollo positivo adolescente. Esta teoría resalta la plasticidad inmanente al desarrollo humano, así como su potencialidad para el cambio sistemático a nivel evolutivo, como fortalezas que, más precisamente, son producto de las influencias mutuas entre el desarrollo de la persona y su acervo biológico, psicológico, ecológico e histórico (Oliva y Pertegal, 2015).

\section{Las actividades estructuradas en el desarrollo positivo adolescente}

En su obra Pedagogía del ocio, Trilla y Rovira (1987) proponen que se diluyan las fronteras entre los tiempos de trabajo y de ocio. Según estos autores, hay que proyectar hacia otras esferas de la actividad humana las características positivas del tiempo libre, de manera que se encuentre en ellas la posibilidad de realización personal, el aprendizaje continuado y conseguir una satisfacción intrínseca en la actividad realizada. Caride (2012) ve también necesario conectar el desarrollo positivo con la educación y el tiempo libre. Este autor va más lejos en el modelo educativo, proponiendo un sistema integrado de tiempos que vertebre la realidad pública y privada del individuo. Es necesario que en este sistema se fomenten valores, actitudes, conocimientos y competencias que ofrezcan alternativas al sistema apresurado de vida que faciliten el disfrute $y$ el ejercicio del ocio. Esto traerá como consecuencia que la realidad que rodea al individuo sea educadora. En concreto, propone que haya un tiempo de calidad en el que implícitamente esté contemplado el ocio y un ingrediente básico: la búsqueda de la felicidad personal. Este tiempo de calidad se ha de caracterizar por promover procesos de maduración pausados y estables, sostenibles y meditados -es decir, profundamente humanos, que den sentido a la propia vida-y, a la vez, por dejar de lado el consumo de experiencias, con emociones intensas y fugaces.

El tiempo de calidad es tiempo libre que, ausente de obligaciones escolares, propicia realizar ac- tividades que satisfacen más (Argyle, 1999), ya sean deportivas, artísticas o recreativas. Los tipos de ocio más satisfactorios son aquellos que son muy similares al trabajo, por tanto, las actividades organizadas suelen producir un impacto más positivo que las no organizadas y aportan más beneficios para el desarrollo y el crecimiento personal que las que no estructuradas (Argyle y Lu, 1992).

Se considera ocio organizado el que realiza un uso constructivo del tiempo a través de actividades. Al promover el desarrollo de los jóvenes (Benson et al., 2012), las actividades poseen tres características: la estructura, la supervisión adulta y el énfasis en la construcción de habilidades (Eccles y Gootman, 2002; Roth y Brooks-Gunn, 2003). Las actividades que integran el ocio estructurado son voluntarias, cuentan con reuniones programadas y regulares, mantienen el desarrollo sobre la base de expectativas y las normas de los participantes, ofrecen supervisión y guía por parte de los adultos y están organizadas en torno al desarrollo particular de las capacidades y el logro de metas, todo lo cual incrementa generalmente su complejidad, a medida que se van desarrollando las habilidades de los participantes. La palabra organizado se utiliza para diferenciar estas actividades de las no estructuradas o de otras formas de pasar el tiempo libre, más pasivas o de cuidados básicos.

La voluntariedad, como criterio definitorio de las actividades de tiempo libre, requiere la propia motivación y la no imposición por la familia, la escuela o el ámbito político. A partir de la alta motivación que suscita una actividad, se produce el estado de flow (Csikszentmilhayi 1996, 2001). Este estado se produce cuando los individuos están completamente sumidos en una actividad, hasta el punto de olvidar la fatiga, el tiempo y todo lo que dicha actividad conlleva. Gracias a la combinación de los dos estados psicológicos: motivación intrínseca y concentración, se produce en el individuo un sentimiento subjetivo agradable, que le hace estar despierto, vivo y abierto a experiencias de desarrollo. 
Las actividades de ocio no estructuradas son aquellas que se llevan a cabo sin reglas formales o sin supervisión de los adultos, presentan pocas metas relacionadas con el desarrollo de habilidades y ocurren de manera relativamente espontánea (por ejemplo, ver la televisión, "pasar el rato" con los compañeros). Todos los adolescentes participan en este tipo de actividades no estructuradas hasta cierto punto. De hecho "pasar el rato" con los amigos es considerada la actividad de tiempo libre preferida de los adolescentes (Van Roosmalen y Krahn, 1996).

Sin embargo, numerosos estudios han indicado que el tiempo que se invierte pasando el rato y la falta de participación en actividades organizadas predicen delincuencia (Yin et al., 1999), problemas de conducta, sintomas de depresión, peores notas escolares (Bartko y Eccles, 2003; McHale et al., 2001), uso de sustancias (Caldwell y Darling, 1999) y juego problemático más frecuente (Moore y Ohtsuka, 200o). Y Mahoney y Stattin (2000) sugirieron que es más probable que los comportamientos antisociales ocurran durante el tiempo libre no estructurado.

Los resultados de abundantes estudios sobre el ocio de los jóvenes, en distintos países, revelan que las actividades de ocio estructuradas se asocian con un desarrollo normativo de los jóvenes, mientras que las actividades de ocio desestructuradas y no supervisadas están vinculadas a conductas de riesgo y a un desarrollo no normativo. Aun así, los jóvenes, en su tiempo libre, suelen combinar actividades estructuradas y no estructuradas (Badura et al., 2018), y también existen actividades de ocio no estructurado que propician el desarrollo.

Una de estas actividades es la lectura por placer, que no reúne las características de actividad estructurada y, en cambio, reporta a los jóvenes un compromiso interno con el aprendizaje (Benson et al., 2012) y una comprensión significativa de las relaciones maduras, los valores personales, la identidad cultural, la seguridad física, las preferencias estéticas y la comprensión del mundo físico, todo lo cual ayuda a los lectores adolescentes en la transición de la niñez a la edad adulta (Howard, 2011).

La importancia de las actividades estructuradas extracurriculares se hace más patente conforme se muestran evidencias sobre la participación en actividades del centro educativo y de la comunidad, ya que proporcionan a los jóvenes actividades a elegir, según sus preferencias, y un amplio abanico de beneficios para un desarrollo positivo (Bundick, 2011; Feldman y Matjasko, 2005; 2007) tales como autoestima, logro académico y profesional, voluntad de ayuda al prójimo, implicación social y capacidad de sobreponerse a la adversidad (Eccles y Barber, 1999; Eccles y Gootman, 2002; Larson, 2000; Marsh y Kleitman, 2002). Según Tieu et al. (2010), aquellos alumnos que participan en actividades más estructuradas tienen mayor autoestima, apoyo social, menos niveles de estrés y, generalmente, un mejor ajuste.

Precisamente, la estructuración de las actividades también se ha relacionado con un descenso de los niveles de violencia y conducta antisocial (Rorie et al., 2011; Vandell y Reisner, 2006) y de consumo de sustancias (Eccles et al., 2003) y con la mejora de la salud (Zambon et al., 2009). Además, estas actividades pueden constituir herramientas útiles, mediante las cuales los niños y adolescentes desarrollan diversas competencias, como la iniciativa (Abbott y Barber, 2007; Larson, 2000), el trabajo en equipo, las habilidades emocionales y un sentido pleno y significativo de su lugar en el mundo (Vandell et al., 2015).

Por su parte, Larson (2000), como pionero en el estudio del desarrollo positivo adolescente en el tiempo libre, a través de los programas extraescolares y comunitarios, destaca el papel de los adultos en el desarrollo del ocio estructurado y propone seis principios que deben regir la misión de los adultos en estas actividades: deben acompañar a los jóvenes en el desarrollo de las actividades; deben impulsar una cultura en la que los jóvenes quieran participar, propiciando un ambiente que promueva la responsabilidad y el liderazgo; deben supervisar las actividades; 
deben facilitar el andamiaje y dar apoyos; deben animar e impulsar a los jóvenes para que se enfrenten a nuevos retos y responsabilidades; y, por último, los adultos deben ajustar los retos a las capacidades de los jóvenes.

Las actividades realizadas por diferentes organizaciones, dentro de un contexto asociativo, favorecen el desarrollo de capacidades y el logro de metas por medio de unas reglas y pautas, todo ello con abundantes ventajas. Ramos et al. (2012) recomiendan la participación de los jóvenes, sobre todo durante la adolescencia tardía, en asociaciones que promuevan actividades organizadas de ocio, ya que a través de ellas se incrementa el apoyo efectivo, el reconocimiento social y la autoestima que genera una ciudadanía activa, con un compromiso y una virtud cívicos (Putnam, 1995).

Las instituciones tienen un papel primordial en el desarrollo positivo de los jóvenes. Por medio del desarrollo de programas de ocio, proporcionan una amplia variedad de actividades y ofrecen espacios que sean acordes con sus preferencias y necesidades individuales (Oropesa et al., 2014). Este es el caso del programa Youth in Europe (ECAD, 2005), iniciado en Islandia, implementado también en otros países y dirigido a la prevención del uso de alcohol en jóvenes, a través de la inversión en actividades de ocio saludable que satisfagan a los adolescentes. Otra iniciativa, propuesta por la Fundación Telefónica, en su convocatoria Thing Big (Makua et al., 2017), ha promovido proyectos de ocio que más interesaban a los jóvenes.

\section{Motivación y satisfacción en el ocio}

Volviendo a las características que definen el ocio para los autores que inician los estudios sobre este tema en Europa, Friedmann (1963) y Dumazedier (1964) señalan que las actividades de ocio deben caracterizarse por la libertad y la satisfacción. La teoría de la autodeterminación (Deci y Ryan, 2000) es un modelo explicativo de la motivación humana aplicable al estudio de las repercusiones que tienen las actividades de tiempo libre en el desarrollo y en el bienestar de los adolescentes. En un estudio llevado a cabo con jóvenes australianos, Garton y Pratt (1991) concluyen que el nivel de motivación de un adolescente tiene una influencia positiva en la satisfacción del ocio. En cuanto a la relación entre la participación en actividades de ocio y la satisfacción, este estudio encontró que la participación medía la relación entre la motivación y la satisfacción. La motivación para el ocio parece jugar un papel clave en la predisposición, lo que podría aumentar o reducir la aceptación de la participación en el ocio y, por lo tanto, la satisfacción con el ocio. En un estudio posterior, realizado con jóvenes taiwaneses, Chen et al. (2013) se encuentran que existe una estrecha relación entre la frecuencia de participación en actividades de ocio y los niveles de interés en las actividades.

\section{La transición a la vida adulta: el binomio ocio-estudio en los adolescentes}

Según Casal et al. (2006), la biografía personal juega un papel fundamental en el itinerario que siguen los jóvenes hacia su vida adulta. En este recorrido las experiencias vitales más significativas se enmarcan en el ocio, como el mundo asociativo, la cultura, la ciudadanía y la relación entre iguales. Merino (2007) afirma que hay competencias útiles para la vida laboral que se adquieren al participar en asociaciones juveniles o en la realización de actividades organizadas de tiempo libre. Este autor también afirma que es necesario analizar las biografías individuales, para establecer la relación causal entre las competencias adquiridas en la transición de la escuela hacia el trabajo.

En la transición hacia la vida adulta hay varios binomios que se deben armonizar para llegar al equilibrio vital y a la madurez: la escuela/casa, vecindario; los pares/la familia; tiempo de trabajo, estudio/tiempo libre. En estos binomios influyen muchas variables exploradas por diversos estudios, como: diferencias de ocio entre carreras técnicas y 
no técnicas (Lemp y Behn, 2008); diversidad en las experiencias de ocio (Olubor y Osunde, 2007); atribuciones personales y autoeficacia (Wise, 2009); ocio para conseguir logros (Patry et al., 2007); y ocio y gestión del tiempo, asociados a los estilos de aprendizaje (García y Santizo, 2010).

Se ha visto que el estudio es la actividad más importante que realizan los jóvenes, cuyo objetivo es el aprendizaje para la vida, a través del desarrollo de competencias enfocadas a la preparación para el futuro laboral. El estudio tiene como objetivo el logro académico y está asociado a determinadas variables: la motivación (Powell y Arriola, 2003; Shim y Ryan, 2005; Tavani y Losh, 2003), la autoestima (DuBois et al., 1998; Owens, 1994), la asertividad y el desarrollo de habilidades sociales (Poyrazli et al., 2002; Thompson y Bundy, 1995) y los enfoques del aprendizaje (Alonso et al., 2012). Como variable negativa está el consumo de sustancias, que se asocia de una manera negativa al rendimiento académico (Poyrazli et al., 2002; Quatman y Watson, 2001).

En el análisis del binomio ocio-estudio se observa que el ocio influye en el rendimiento académico-asociado a otros factores, como la personalidad y las relaciones interpersonales-y que el rendimiento académico depende de ellos, por lo que un buen ocio favorece un buen trabajo (Caso y Hernández, 2007).

En el otro extremo del binomio, también el modo en que se realiza el aprendizaje influye en el ocio, y un buen ocio se asocia con las competencias desarrolladas a través del desempeño académico. Según la teoría de Biggs (1987), el enfoque de aprendizaje es la manera habitual en la que un estudiante adquiere logros académicos y resulta de la interrelación de tres elementos clave (Biggs, 1993): la motivación, el proceso o estrategia que utiliza y el logro académico. Según López et al. (2016), el estudio de modelos de aprendizaje debe tener en cuenta las variables de enfoque profundo y compromiso. Para estos autores:
1) la influencia del rendimiento académico anterior es predictor de un enfoque profundo, orientado a la consecución de significado y compresión, opuesto al enfoque superficial, caracterizado por la no profundización y reproducción de los conocimientos; 2) los valores más altos de compromiso se asocian con valores más altos de enfoque profundo y viceversa, por lo que se puede afirmar que los enfoques de aprendizaje y el compromiso son predictores recíprocos; 3 ) el compromiso elevado y el enfoque profundo se correlacionan con una disponibilidad menor de tiempo libre y ocio. Este último resultado invita a estudiar la calidad de las actividades en relación con el tiempo libre disponible.

\section{Conclusión}

Desde el origen de nuestra cultura, el ocio se ha visto como fuente de realización personal y libertad, pero ha sido a partir del siglo XX cuando su impacto ha sido más evidente en la sociedad. En los jóvenes el ocio juega un papel primordial y es necesario avanzar en un modelo de ocio positivo que promueva el ejercicio de competencias, el fortalecimiento del carácter y recursos activos, tanto a nivel individual como interpersonal. En este modelo son importantes el propósito de vida, la iniciativa y el sentido de coherencia. La meta es alcanzar un tiempo de calidad, en el que sea posible la satisfacción personal y la felicidad. El ocio estructurado, como uso constructivo del tiempo, a través de la realización de actividades, potencia su desarrollo óptimo. El estudio, actividad principal de muchos jóvenes, debe armonizarse con el valor en alza del ocio, para poder culminar con éxito su transición hacia la vida adulta.

Es preciso que haya más investigaciones sobre las actividades de ocio estructuradas y no estructuradas de los adolescentes, que analicen la relación entre estos dos modelos de actividades, con el fin de proponer nuevos ámbitos de desarrollo personal y una mejora de la calidad de su ocio. 


\section{Referencias}

Anderson, N. (1961). Work and leisure. Taylor \& Francis.

Abbott, B. D. y Barber, B. L. (2007). Not just idle time: Adolescents' developmental experiences provided by structured and unstructured leisure activities. The Australian Educational and Developmental Psychologist, 24(1), 59-81. https://doi.org/10.1017/So816512200029102

Alonso, A. I., López-Aguado, M., González, I., y Fernández, E. (2012). El ocio y los enfoques de aprendizaje en estudiantes universitarios de Enfermería. Revista de Investigación Educativa, 30(1), 53-70. https://doi. org/10.6018/rie.30.1.111581

André, J. M. (1966). L'otium dans la vie morale et intellectuelle romaine: des origines à l'époque augustéenne. PUF.

Antonovsky, A. (1987). Unraveling the mystery of health. How people manage stress and stay well. San Fransisco: Jossey-Bass.

Antonovsky, A. (1993a). Complexity, conflict, chaos, coherence, coercion and civility. Social Science \& Medicine, 37(8), 969-974. https://doi.org/10.1016/0277-9536(93)90427-6

Antonovsky, A. (1993b). The structure and properties of the sense of coherence scale. Social Science \& Medicine, 36(6), 725-733. https://doi.org/10.1016/0277-9536(93)90033-Z

Argyle, M. y Lu, L. (1992). New directions in the psychology of leisure. The New Psychologist, 1(3), 5-11. http://web. ba.ntu.edu.tw/luolu/New\%2odirections\%2oin\%2othe\%2opsy chology\%20of\%2oleisure.pdf

Argyle. M. (1999). Causes and correlates of happiness. En D. Kahneman, E. Diener y N. Schwarz (eds.), Well-being: the foundations of hedonic psychology (pp. 353-373). Russell Sage Foundation.

Badura, P., Madarasova Geckova, A., Sigmundova, D., Sigmund, E., Van Dijk, J. P. y Reijneveld, S. A. (2018). Can organized leisure-time activities buffer the negative outcomes of unstructured activities for adolescents' health? International Journal of Public Health, 63(6), 743-751. http://doi.org/10.1007/s00038-018-1125-3

Bartko, W. T. y Eccles, J. S. (2003). Adolescent participation in structured and unstructured activities: A personoriented analysis.Journal of Youth and Adolescence,32(4), 233-241. http://doi.org/10.1023/A:1023056425648

Baudrillard, J. (2009). La sociedad de consumo: sus mitos, sus estructuras. Siglo XXI.

Benson, P. L., Leffert, N., Scales, P. C. y Blyth, D. A. (2012). Beyond the "village" rhetoric: Creating healthy communities for children and adolescents. Applied Developmental Science, 16(1), 3-23. https://doi.org/10.1207/ S1532480xadsO2O3_3

Bernal Guerrero, A. y König Bustamante, K. L. (2017). Percepciones de adolescentes sobre la educación según la identidad personal. Revista Española de Pedagogía, 75(267), 181-198. http://doi.org/10.22550/REP75-2-2017-01 
Biggs, J. B. (1987). Student approaches to learning and studying. [Research Monograph] Australian Council for Educational Research.

Biggs, J. (1993). What do inventories of students' learning processes really measure? A theoretical review and clarification. British Journal of Educational Psychology, 63(1), 3-19. https://doi.org/10.1111/j.2044-8279.1993. tbo1038.x

Bundick, M. J. (2011). Extracurricular activities, positive youth development, and the role of meaningfulness of engagement. Journal of Positive Psychology, 6(1), 57-74. http://dx.doi.org/10.1080/17439760.2010.536775

Cacérès, B. (1973). Loisirs et travail: du Moyen Age à nos jours. Seuil.

Caldwell, L. L.y Darling, N. (1999). Leisure context, parental control, and resistance to peer pressure as predictors of adolescent partying and substance use: An ecological perspective. Journal of Leisure Research, 37(1), 5777. http://doi.org/10.1080/00222216.1999.11949851

Caride, J. A. (2012). Lo que el tiempo educa: el ocio como construcción pedagógica y social.Arbor, 188(754), 301-313. https://doi.org/10.3989/arbor.2012.754n2004

Casal, J., García, M., Merino Pareja, R.y Quesada, M. (2006). Aportaciones teóricas y metodológicas a la sociología de la juventud desde la perspectiva de la transición. Papers: Revista de Sociología, 79, 21-48. http://doi. org/10.5565/rev/papers/v79no.798

Caso-Niebla, J. y Hernández, L. (2007). Variables que inciden en el rendimiento académico de adolescentes mexicanos. Revista Latinoamericana de Psicología, 39(3), 487-501. https://www.redalyc.org/pdf/805/80539304.pdf

Chen, Y. C., Li, R. H.y Chen, S. H. (2013). Relationships among adolescents' leisure motivation, leisure involvement, and leisure satisfaction: A structural equation model. Social Indicators Research, 110(3), 1187-1199. http:// doi.org/10.1007/s11205-011-9979-2

Codina, N. (2007). Psicología social del ocio y del tiempo libre: Su constante quehacer y su carácter "fuzzy". En M. J. Monteagudo Sánchez (ed.), El ocio en la investigación actual: Una lectura desde ámbitos, disciplinas, grupos de población y contextos geográficos (pp. 157-173). Universidad de Deusto.

Coleman, J. C. (1978). Current contradictions in adolescent theory. Journal of Youth and Adolescence, 7(1), 1-11. http://doi.org/10.1007/BF01538683

Csikszentmihalyi, M. (1975). Beyond boredom and anxiety. Jossey-Bass.

Csikszentmihalyi, M. (1996). Flow and the psychology of discovery and invention. Harper Collins.

Csikszentmihalyi, M. (2001). Ocio y creatividad en el desarrollo humano. En M. Csikszentmihalyi, M. Cuenca, C. Buarque y V. Trigo (Eds.), Ocio y desarrollo. Potencialidades del ocio para el desarrollo humano (pp. 17-32). Universidad de Deusto. 
Cuenca Cabeza, M. y Goytia Prat, A. (2012). Ocio experiencial: antecedentes y características. Arbor, 188(754), 265281. http://dx.doi.org/10.3989/arbor.2012.754n2001

Damon, W. (2009). The path to purpose: How young people find their calling in life. Simon \& Schuster.

De Grazia, S. (1966). Tiempo, trabajo y ocio. Tecnos.

Deci, E. L.y Ryan, R. M. (eds.) (2002). Handbook of self-determination research. I University Rochester Press.

Deci, E. L.y Ryan, R. M. (2000). The "what" and "why" of goal pursuits: Human needs and the self-determination of behavior, Psychological Inquiry, 11(4), 227-268. http://dx.doi.org/10.1207/S15327965PLl1104_01

Devine, M. A. y Parr, M. G. (2008). "Come on in, but not too far:" Social capital in an inclusive leisure setting. Leisure Sciences, 30(5), 391-408. http://dx.doi.org/10.1080/01490400802353083

DuBois, D. L., Bull, C. A., Sherman, M. D. y Roberts, M. (1998). Self-esteem and adjustment in early adolescence: A social-contextual perspective. Journal of Youth and Adolescence, 27(5), 557-583. http://doi. org/10.1023/A:1022831006887

Dumazedier, J. (1964). Hacia una civilización del ocio. Estela.

Dumazedier, J. (1971). Realidades del ocio e ideologías. En Dumazedier et al., Ocio y sociedad de clases. Fontanella.

Eccles, J. S. y Barber, B. L. (1999). Student council, volunteering, basketball or marching band: What kind of extracurricular involvement matters? Journal of Adolescent Research, 14, 10-43. http://doi. org/10.1177/0743558499141003

Eccles, J.y Gootman, J. (eds.) (2002). Community programs to promote youth development. National Academy Press.

Eccles, J. S., Barber, B. L., Stone, M.y Hunt, J. (2003). Extracurricular activities and adolescent development. Journal of Social Issues, 59, 865-89o. http://doi.org/10.1007/s10964-010-9563-0

ECAD - European Cities against Drugs (2005). Youth in Europe - A Drug Prevention Programme. [Portal web] http://www.ecad.net/activ/Youth_Programme.pdf

Erikson, E. H. (1968). Identity: Youth and crises. Norton.

Feldman, A. F. y Matjasko, J. L. (2005). The role of school-based extracurricular activities in adolescent development: A comprehensive review and future directions. Review of Educational Research, 75(2), 159-210. https://doi.org/10.3102/00346543075002159

Feldman, A. F.y Matjasko, J. L. (2007). Profiles and portfolios of adolescent school-based extracurricular activity participation. Journal of Adolescence, 30(2), 313-332. http://doi.org/10.1016/j.adolescence.2006.03.004

Frederic, M. (1985). Psicosociología del tiempo libre. Un enfoque crítico. Trillas. 
ISSN 0123-1294 | e-ISSN 2027-5358 | Educ.Educ. Vol. 23. No. 2 | Mayo-julio de 2020 | pp. 201-220

Universidad de La Sabana | Facultad de Educación

Friedmann, G. (1956). Problemas humanos del maquinismo industrial. Sudamericana.

Friedmann, G. (1963). Tratado de sociología del trabajo. Fondo de Cultura Económica.

García-Cué, J. L. y Santizo, J. A. (2010). Análisis de la relación entre la gestión del tiempo libre, el ocio y los estilos de aprendizaje. Journal of Learning Styles, 3(5). http://revistaestilosdeaprendizaje.com/article/view/899

Garton, A. F. y Pratt, C. (1991). Leisure activities of adolescent school students: Predictors of participation and interest. Journal of Adolescence, 14(3), 305-321. https://doi.org/10.1016/0140-1971(91)90023-K

Glover, T. D. y Hemingway, J. L. (2005). Locating leisure in the social capital literature. Journal of Leisure Research, 37(4),387-401. https://doi.org/10.1080/00222216.2005.11950059

Harré,R.(1990).Leisureanditsvarieties. LeisureStudies, 9(3),187-195.https://dx.doi.org/10.1080/02614369000390161

Havighurst, R. J. (1961). The nature and values of meaningful free-time activity. En R. W. Kleemeier (ed.), Aging and leisure (pp. 309-344). Oxford University Press.

Howard, V. (2011). The importance of pleasure reading in the lives of young teens: Self-identification, self-construction and self-awareness. Journal of Librarianship and Information Science, 43(1), 46-55. https://doi. org/10.1177/og61000610390992

Iso-Ahola, S. E. (1980). The social psychology of leisure and recreation. W.C. Brown.

INJUVE (2015). Jóvenes, ocio y consumo. [Portal institucional] Ministerio de Derechos Sociales y Agenda, España. http://www.injuve.es/observatorio/ocio-y-tiempo-libre/jovenes-ocio-y-consumo

Kaplan, M. (1960). Leisure in America. A social inquiry. Wiley.

Kristjansson, A. L., Sigfusdottir, I. D., Thorlindsson, T., Mann, M. J., Sigfusson, J. y Allegrante, J. P. (2015). Population trends in smoking, alcohol use and primary prevention variables among adolescents in Iceland, 19972014. Addiction, 111(4), 645-652. https://doi.org/10.1111/add.13248

Kroger, J. (1993). The role of historical context in the identity formation process of late adolescence. Youth \& Society, 24(4), 363-376. http://dx.doi.org/10.1177/0044118X93024004003

Langenhove, L. (1992). The identification and measurement of psy chological aspects of leisure. Leisure Studies, 11(2), 147-156. https://dx.doi.org/10.1080/02614369200390051

Larson, R. W. (2000). Toward a psy chology of positive youth development. American Psychologist, 55(1), 170-183. http://dx.doi.org/10.1037/0003-066X.55.1.170

Lemp, M.y Behn, V. (2008). Utilización del tiempo libre de estudiantes del área de salud de la Institución Educacional Santo Tomás, Talca. Ciencia y Enfermeria, 14(1),53-62.https://scielo.conicyt.cl/scielo.php?script=sci_ arttext\&pid=S0717-95532008000100007 
Lerner, R. M. (2004). Liberty: Thriving and civic engagement among America's youth. Sage.

Lerner, R. M. (2006). Developmental science, developmental systems, and contemporary theories of human development. En W. E. Damon y R. M. Lerner (eds.), Handbook of Child Psychology: Theoretical models of human development (vol. 1, pp. 1-17). John Wiley \& Sons.

López-Alonso, A. I., López-Aguado, M., Fernández-Martínez, M. E., Liébana, C. y Gutiérrez-Provecho, L. (2016). Los enfoques de aprendizaje, el "engagement", el ocio y el rendimiento anterior. Propuesta de un modelo. Bordón. Revista de Pedagogía, 68(4), 67-88.https://doi.org/10.13042/Bordon.2016.40940

Lynd, R. S.y Lynd, H. M. (1929). Middletown; a study in contemporary American culture. Harcourt Brace.

Mahoney, J. L. y Stattin, H. (2000). Leisure activities and adolescent antisocial behavior: The role of structure and social context. Journal of Adolescence, 23(2), 113-127. https://doi.org/10.1006/jado.2000.0302

Makua, A., Cuenca-Amigo, M. y Del Valle, R. S. (2017). Relación entre las prácticas de ocio significativas de la juventud y el emprendimiento social. El caso Think Big Jóvenes. OBETS. Revista de Ciencias Sociales, 12 (extra 1), 151-176. https://doi.org/10.14198/OBETS2017.12.1.16

Mannheim, K. (1953). Libertad, poder y planificación democrática. Fondo de Cultura Económica.

Marsh, H. W. y Kleitman, S. (2002). Extracurricular school activities: The good, the bad, and the non-linear. Harvard Educational Review, 72, 464-514. http://dx.doi.org/10.17763/haer.72.4.051388703v7v7736

McHale, S. M., Crouter, A. C. y Tucker, C. J. (2001). Free-time activities in middle childhood: Links with adjustment in early adolescence. Child Development, 72(6), 1764-1778. https://doi.org/10.1111/1467-8624.00377

Mead, M. (1957). The pattern of leisure in contemporary American culture. The Annals of the American Academy of Political and Social Science, 313(1), 11-15. https://doi.org/10.1177/000271625731300105

Merino, R. (2007). Pathways from school to work: can the competences acquired in leisure activities improve the constructionofpathways? JournalofEducationandWork, 20(2),139-159.https://doi.org/10.1080/13639080701314696

Mills, C. W. (1951). White collar: The American middle classes (Vol. 3). Oxford University Press.

Moore, S.y Ohtsuka, K. (2000). The structure of young people's leisure and their gambling behaviour. Behaviour Change, 17(3), 167-177. https://doi.org/10.1375/bech.17.3.167

Munné, F. (1980). Psicosociología del tiempo libre: un enfoque crítico. Trillas.

Neulinger, J. (1974) “The Psychology of Leisure.” Research approaches to the study of leisure. Charles C. Thomas.

Oliva, A. y Pertegal, M. A. (2015). El desarrollo positivo adolescente. Un nuevo paradigma para la investigación y la intervención. En A. Oliva (ed.), Desarrollo positivo adolescente (pp. 19-40). Síntesis. 
Olubor, R. O. y Osunde, U. A. (2007). Analysis of personal study time and leisure among university undergraduates in southern Nigeria. College Student Journal, 41(2), 352-365. https://www.questia.com/ read/1G1-163679004/analysis-of-personal-study-time-and-leisure-among

Oropesa, F. (2014). La influencia del tiempo libre en el desarrollo evolutivo adolescente. Apuntes de Psicología, 32(3), 235-244. https://hdl.handle.net/11441/85215

Oropesa, F., Moreno, C., Pérez, P. y Muñoz-Tinoco, V. (2014). Routine leisure activities: Opportunity and risk in adolescence/Rutinas de tiempo libre: oportunidad y riesgo en la adolescencia. Cultura y Educación, 26(1), 159-183. https://doi.org/10.1080/11356405.2014.908670

Owens, T. J. (1994). Two dimensions of self-esteem: Reciprocal effects of positive self-worth and self-depreciation on adolescent problems. American Sociological Review, 59(3), 391-407. https://doi.org/10.2307/2095940

Patry, D.A., Blanchard, C.M.y Mask, L. (2007). Measuring university students' regulatory leisure coping styles: Plannedbreathersoravoidance? LeisureSciences, 29(3),247-265.https://dx.doi.org/10.1080/01490400701257963

Peterson, C. y Seligman, M. E. (2004). Character strengths and virtues: A handbook and classification (vol. 1). Oxford University Press.

Peterson, C. (2006). The Values in Action (VIA) classification of strengths. En M. Csikszentmihalyi e I. Csikszentmihalyi (eds.). A life worth living: Contributions to positive psychology (pp. 29-48). Oxford University Press.

Portes, A. (1998). Social capital: Its origins and applications in modern sociology. Annual Review of Sociology, 24(1), 1-24. https://doi.org/10.1146/annurev.soc.24.1.1

Poyrazli, S., Arbona, C., Nora, A., McPherson, R.y Pisecco, S. (2002). Relation between assertiveness, academic selfefficacy, and psychosocial adjustment among international graduate students. Journal of College Student Development, 43(5), 32-42. https://eric.ed.gov/?id=EJ653328

Powell, C. L. y Arriola, K. R. (2003). Relationship between psychosocial factors and academic achievement among African American students. Journal of Educational Research, 96(3), 175-181. https://dx.doi. org/10.1080/00220670309598805

Puig, J. M. y Trilla, J. (1987). Pedagogía del ocio. Laertes.

Putnam, R. D. (1995). Bowling alone: America's declining social capital. Journal of Democracy, 6(1), 65-78. https:// doi.org/10.1007/978-1-349-62397-6_12

Quatman, T. y Watson, C. M. (2001). Gender differences in adolescent self-esteem: An exploration of domains. The Journal of Genetic Psychology, 62(1), 93-117. https://dx.doi.org/10.1080/00221320109597883

Ramos,P.,Rivera,F.yMoreno,C.(2012).Beneficios delcontextoasociativoenlas actividades detiempolibredelosadolescentes españoles. Infancia y Aprendizaje, 35(3), 365-378. https://dx.doi.org/10.1174/021037012802238984

Riesman, D. (1968). La muchedumbre solitaria. Paidós. 
Riesman, D., Denney, R. y Glazer, N. (1961). The lonely crowd. A study of the changing American character. Yale University Press.

Riesman, D. (1964). Abundance for what? Doubleday.

Rifkin, J. (1995). The end of work: The decline of the global labor force and the dawn of the post-market era. Putnam.

Rivera, F., Ramos, P., Moreno, C. y Hernán, M. (2011). Análisis del modelo salutogénico en España: aplicación en salud pública e implicaciones para el modelo de activos en salud. Revista Española de Salud Pública, 85(2), 129-139. https://www.redalyc.org/articulo.oa?id=17020112002

Rodríguez Suárez, J. y Agulló Tomás, E. (2002). Psicología social y ocio: Una articulación necesaria. Psicothema, 14(1), 124-133. http://hdl.handle.net/10651/26895

Romo, C. (2003). Tiempo de libertad para la libertad. Algunos apuntes sobre la crítica a la alienación del ocio en la sociedad industrial avanzada. BAETICA. Estudios de Historia Moderna y Contemporánea, 25, 773-79o. https://revistas.uma.es/index.php/baetica/article/view/394

Rorie, M., Gottfredson, D. C., Cross, A., Wilson, D. y Connell, N. M. (2011). Structure and deviancy training in afterschool programs. Journal of Adolescence, 34(1), 105-107. https://doi.org/10.1016/j.adolescence.2010.01.007

Roth, J. L. y Brooks-Gunn, J. (2003). What exactly is a youth development program? Answers from research and practice. Applied Developmental Science, 7(2), 94-111. https://doi.org/10.1207/S1532480XADSO702_6

Shim, S. y Ryan, A. (2005). Changes in self-efficacy, challenge avoidance, and intrinsic value in response to grades: The role of achievement goals. Journal of Experimental Education, 73(4), 333-349. https://dx.doi. org/10.3200/JEXE.73.4.333-349

Siegrist, J. (1993). Sense of coherence and sociology of emotions. Social Science \& Medicine, 37(8), 978-979. https://doi.org/10.1016/0277-9536(93)90430-c

Stebbins, R. A. (1982). Serious leisure a conceptual statement. Sociological Perspectives, 25(2), 251-272. https://doi. org/10.2307/1388726

Stebbins, R. A. (1992). Amateurs, professionals, and serious leisure. Montreal: McGill-Queen's Press.

Stebbins, R. A. (2012). Compromiso temporal discrecional: efectos sobre la elección y el estilo de ocio. Arbor, 188(754), 293-300. https://doi.org/10.3989/arbor.2012.754n2003

Tavani, C. M. y Losh, S. C. (2003). Motivation, self-confidence, and expectations as predictors of the academic performances among our high school students. Child Study Journal, 33(3), 141-151. https://go.gale.com/ps/ anonymous?id=GALE\%7CA116924600\&sid=googleScholar\&v=2.1\&it=r\&linkaccess $=$ abs \&issn=0009400 $5 \& p=A O N E \& S W=W$

Thompson, K. y Bundy, K. (1995). Social skills training for young adolescents: Symbolic and behavioral components. Adolescence, 30(119), 723-735. https://search.proquest.com/openview/507cd498abcd7d17537baa8b2 b677cod/1?pq-origsite $=$ gscholar $\& c b l=41539$ 
Tinsley, H. E. A. y Tinsley, D. J. (1986). A theory of the attributes, benefits, and causes of leisure experience. Leisure Sciences, 8, 1-45. https://dx.doi.org/10.1080/01490408609513056

Tieu, T.-T., Pancer, S. M., Pratt, M. W., Birnie-Lefcovitch, S., Wintre, M. G., Poliv J. y Adams, G. (2010). Helping out or hanging out: The features of involvement and how it relates to university adjustment. Journal of Higher Education, 60, 343-355. https://doi.org/10.1007/s10734-009-9303-0

Van Roosmalen, E. y Krahn, H. (1996). Boundaries of youth. Youth \& Society, 28(1), 3-39. https://doi. org/10.1177/0044118X96028001001

Vandell, D. L.y Reisner, E. R. (2006). Developmental outcomes associated with the after-school contexts of lowincome children and youth. En H. B. Weiss, Critical issues in adolescent participation in out-of-school time activities. [Simposio dirigido] Biennial Meeting of the Society for Research in Adolescence, San Francisco.

Vandell, D. L., Larson, R., Mahoney, J. y Watts, T. (2015). Children's organized activities. En M. H. Bornstein y T. Leventhal (eds.), Handbook of child psychology and developmental science. Ecological settings and processes in developmental systems (vol. 4). Wiley Interscience.

Veblen, T. (2005). Teoría de la clase ociosa. Fondo de Cultura Económica.

Weber, M. (2012). La ética protestante y el espiritu del capitalismo. Fondo de Cultura Económica.

Wise, J. B. (2009). Using the knowledge-and-appraisal personality architecture to predict physically active leisure self-efficacy in university students. Journal of Applied Social Psychology, 39(8), 1913-1927. https://doi. org/10.1111/j.1559-1816.2009.00509.x

WLRA - World Leisure and Recreation Association (1994). WLRA International Charter for Leisure Education. ELRA - European Leisure and Recreation Association, Summer, 13-16.

Wolfenstein, M. (1955). Fun morality: An analysis of recent child-training literature. En M. Mead y M. Wolfenstein (eds.), Childhood in contemporary cultures (pp.168-178). University of Chicago Press.

Yin, Z., Katims, D. S. y Zapata, J. T. (1999). Participation in leisure activities and involvement in delinquency by Mexican American adolescents. Hispanic Journal of Behavioral Sciences, 21(2), 170-185. https://doi. org/10.1177/0739986399212004

Zambon, A., Morgan, A., Vereecken, C., Colombini, S., Boyce, W., Mazur, J., Lemma, P.y Cavallo, F. (2009). The contribution of club participation to adolescent health: evidence from six countries. Journal of Epidemiology \& Community Health, 64(01), 89-95. https://doi.org/10.1136/jech.2009.088443 